\title{
Compressibility of $\mathrm{Fe}_{1.087} \mathrm{Te}$ : A High-Pressure X-ray Diffraction Study
}

\author{
J.-E. Jørgensen, ${ }^{*}$ J. Staun Olsen ${ }^{2}$ and L. Gerward ${ }^{3}$ \\ ${ }^{1}$ Department of Chemistry, University of Aarhus, DK-8000 Aarhus C, Denmark; ${ }^{2}$ Niels Bohr \\ Institute, Oersted Laboratory, DK-2100 Copenhagen, Denmark, ${ }^{3}$ Department of Physics, Technical \\ University of Denmark, DK-2800 Lyngby, Denmark;
}

\begin{abstract}
$\mathrm{Fe}_{1.087} \mathrm{Te}$ exhibits three phases in the pressure range from ambient to $16.6 \mathrm{GPa}$ and becomes amorphous at higher pressures. All three phases have tetragonal symmetry. The low pressure Tphase is stable in the pressure range $0 \leq P<4.1 \mathrm{GPa}$ and found to be relatively soft having zero pressure bulk modulus $B_{0}=36(1) \mathrm{GPa}$. The intermediate cT-phase is less compressible with $B_{0}=$ 88(5) GPa and stable in the pressure range $4.1 \leq P<10 \mathrm{GPa}$ while a more compressible phase was observed between 10 and $16.6 \mathrm{GPa}$.
\end{abstract}

PACS codes: 61.10.-i, 62.50.+p, 74.70.-b

Keywords: A. Superconductors, C. X-ray scattering, E. High pressure

*Corresponding author 


\section{Introduction}

Iron containing pnictide and chalcogenide superconducting compounds have recently attracted much attention, and superconductivity in $\mathrm{FeSe}_{1-\mathrm{x}}$ with $T_{\mathrm{c}}=8 \mathrm{~K}$ was discovered shortly after the surprising discovery of the FeAs-based superconductors such as $\operatorname{LaFeAs}\left(\mathrm{O}_{1-\mathrm{x}} \mathrm{F}_{\mathrm{x}}\right)$ with superconducting transition temperatures $T_{\mathrm{c}}$ up to $55 \mathrm{~K}$ [1-3]. Later, a huge enhancement of the superconducting transition temperature was observed in a tetragonal FeSe superconductor under high pressure. A pressure of $1.48 \mathrm{GPa}$ enhanced the onset of $T_{\mathrm{c}}{ }^{\text {onset }}$ and $T_{\mathrm{c}}{ }^{\text {zero }}$ to 27 and $13.5 \mathrm{~K}$, respectively [4]. Both the FeAs-based superconductors and the tetragonal $\mathrm{FeSe}_{1-\mathrm{x}}$ contain $\mathrm{FeX}(\mathrm{X}=$ As or Se) layers composed of edge-sharing $\mathrm{FeX}_{4}$ tetrahedra. The $\beta$-phase $\mathrm{Fe}_{1+\mathrm{x}} \mathrm{Te}$ and $\mathrm{FeSe}_{1-\mathrm{x}}$ are isostructural having the $\alpha-\mathrm{PbO}$ type structure (space group $P 4 / \mathrm{nmm} \# 129$ ) with the iron chalcogenide layers stacked along the $c$-axis [5]. The FeTe phase is non-superconducting but both FeTe and FeSe have 14 valence electrons per formula unit (and therefore also per FeX layer) which is the same valence electron count as for the single negatively charged FeAs layers in undoped iron pnictides such as $\mathrm{BaFe}_{2} \mathrm{As}_{2}$ and $\mathrm{LaFeAsO}$ type compounds, and FeTe should therefore be viewed as a reference material for the superconducting iron pnictides. Mizucuchi et al. [6] have suggested FeTe as a candidate for a new iron-based superconductor. They studied the pressure dependence of the resistivity of this compound, and superconductivity was actually observed in Se and S doped FeTe. $\mathrm{Fe}\left(\mathrm{Se}_{1-\mathrm{x}} \mathrm{Te}_{\mathrm{x}}\right)_{0.82}$ has been shown to be superconducting with a maximum $T_{c}$ of $14 \mathrm{~K}$ for $\mathrm{x} \approx$ 0.6 , and $T_{c}$ was found to decrease rapidly for $\mathrm{x}>0.9$ [7] while a $T_{c}$ of $8 \mathrm{~K}$ was observed for $\mathrm{Fe}_{1.06} \mathrm{Te}_{0.88} \mathrm{~S}_{0.14}$ [8]. Ipser et al. have studied the Fe-Te phase diagram [9]. The $\beta$-FeTe phase was found to be non-stoichiometric with a stability range from $\mathrm{Fe}_{1.07} \mathrm{Te}$ to $\mathrm{Fe}_{1.19} \mathrm{Te}$ at ambient temperature. Later, Okamatoto [10] found a slightly different stability range $\left(\mathrm{Fe}_{1.04} \mathrm{Te}\right.$ to $\left.\mathrm{Fe}_{1.08} \mathrm{Te}\right)$. Grønvold et al. [11] first suggested the excess iron atoms in the $\beta$-FeTe phase be located in partially occupied interstitial sites.

The interplay between magnetism and superconductivity in the ferropnictides has been studied in detail, and it has been shown that doping suppresses magnetic ordering as well as structural transitions to lower symmetry, thereby stabilizing the superconducting state. $\mathrm{Fe}_{1.125} \mathrm{Te}$ shows several similarities with the undoped parent compounds of the superconducting ferropnictides. It has been shown to order into a commensurate antiferromagnetic structure with $T_{N} \approx 70 \mathrm{~K}$, the magnetic ordering process being accompanied by a tetragonal to monoclinic phase transition [5]. 
This result was recently confirmed in a neutron diffraction study of $\mathrm{Fe}_{1.076} \mathrm{Te}$, which also showed commensurate antiferromagnetic ordering and a simultaneous transition to monoclinic symmetry [12]. It was furthermore shown that the low temperature crystal and magnetic structures depend on the Fe:Te ratio as $\mathrm{Fe}_{1.141}$ Te was shown to transform to orthorhombic symmetry (space group Pmmn \#59) at $63 \mathrm{~K}$ and to undergo an incommensurate antiferromagnetic ordering. However, despite the similarity between the FeX layers in $\beta$-FeTe, $\mathrm{BaFe}_{2} \mathrm{As}_{2}$ and the $\mathrm{LaFeAs}\left(\mathrm{O}_{1-\mathrm{x}} \mathrm{F}_{\mathrm{x}}\right)$ type compounds, their antiferromagnetic structures are different as they have different propagation vectors and directions of the magnetic moments of the iron atoms [13].

Takahasi et al. [14] recently studied the low temperature magnetic and electrical properties of $\mathrm{FeTe}_{0.92}\left(\mathrm{Fe}_{1.087} \mathrm{Te}\right)$ under pressure, reporting a series of pressure-induced structural transitions. The first low-temperature high-pressure phase HP1 appears at $1 \mathrm{GPa}$ and below $65 \mathrm{~K}$. It extends up to 2.3 GPa where the second low-temperature high-pressure phase HP2 becomes stable. It was furthermore suggested that a new magnetic phase becomes stable within the HP1 phase and that the commensurate antiferromagnetic ordering is suppressed at pressures higher than $1.5 \mathrm{GPa}$. No indication of pressure-induced superconductivity was observed in this study and the stability of the HP2 was found to increase with increasing pressure.

The aim of the present experiment is to investigate the high-pressure behaviour of $\mathrm{Fe}_{1.087} \mathrm{Te}$ and to observe possible pressure-induced phase transitions in a pressure range up to $20 \mathrm{GPa}$.

\section{Experimental}

$\mathrm{Fe}_{1.087} \mathrm{Te}$ was prepared from a 1.087:1 mixture of iron and tellurium powders. The powder mixture was placed in a quartz ampoule, which was closed under vacuum and heated at a rate of $200{ }^{\circ} \mathrm{C} / \mathrm{h}$ to $780{ }^{\circ} \mathrm{C}$ and kept at this temperature for 10 hours. X-ray powder diffraction data at ambient conditions were recorded on a Bruker D8 powder diffractometer using $\mathrm{Cu} \mathrm{K}_{\alpha 1}$ radiation $(\lambda=$ $1.54039 \AA$ ). Fluorescence radiation from iron was suppressed by the use of a Sol-X energy discriminating detector.

High-pressure X-ray powder patterns were recorded using synchrotron radiation and the whitebeam energy-dispersive method at the Hamburg Synchrotron Radiation Laboratory (HASYLAB) 
using radiation in the $10-60 \mathrm{keV}$ photon energy range from a bending magnet. The experiment was performed at station F3 using an unfocused X-ray beam defined by two pairs of crossed slits. The slit width was $60 \mu \mathrm{m}$ and the scattered photons were detected by a high purity Ge solid state detector. Further details of the diffractometer, working in the energy-dispersive mode, has been described elsewhere by Olsen [15]. High pressures were obtained using a Syassen-Holzapfel type diamond-anvil cell [16]. The diameter of the anvil cullets were $0.6 \mathrm{~mm}$ and the sample and a small ruby chip were enclosed in a hole of diameter $0.2 \mathrm{~mm}$ in an Inconel gasket preindented to $60 \mu \mathrm{m}$. A 4:1 ethanol/methanol mixture was used as pressure-transmitting medium, and the pressure was determined from the wavelength shift of the ruby line, applying the non-linear pressure scale of Mao et al. [17]. The uncertainty in the pressure determination is estimated at $0.1 \mathrm{GPa}$ for pressures below $10 \mathrm{GPa}$. For higher pressures the uncertainty may be larger because of possible deviations from hydrostatic conditions. The Bragg angle $\left(2 \theta=7.81^{\circ}\right)$ associated with each experimental run was deduced from a zero-pressure spectrum of rocksalt $(\mathrm{NaCl})$ with a known lattice constant in the pressure cell.

\section{Results and discussion}

$\mathrm{Fe}_{1.087} \mathrm{Te}$ was studied by X-ray powder diffraction at ambient conditions. The structural model was refined in space group P4/nmm with the iron and tellurium atoms Fel and Te located in the fully occupied sites $2 a\left(3 / 4 \frac{1}{4} 0\right)$ and $2 c\left(1 / 4 \frac{1}{4} z_{\mathrm{Te}}\right)$ positions, respectively [18]. Two sites in space group $P 4 / \mathrm{nmm}$ are in principle available for the excess iron atoms $\mathrm{Fe} 2$, the $2 c\left(1 / 41 / 4 z_{\mathrm{Fe} 2}\right)$ and $2 b(3 / 41 / 41 / 2)$ sites. However, trial refinements with the excess Fe 2 atoms located in the tetrahedraly coordinated $2 b\left(3 / 4 \frac{1}{4} \frac{1}{2}\right)$ position indicated that this position is unoccupied and subsequent refinements were done with the excess $\mathrm{Fe} 2$ atoms located in the $2 c\left(1 / 4 \frac{1}{4} z_{\mathrm{Fe} 2}\right)$ position. The lattice parameters were determined to $a=3.82331(7) \AA$ and $c=6.2924(1) \AA$ corresponding to a unit-cell volume of 91.980(3) $\AA^{3}$ and the refineable coordinates of $\mathrm{Fe} 2$ and Te refined to $z_{\mathrm{Fe} 2}=0.688(2)$ and $z_{\mathrm{Te}}=$ $0.2807(3)$. The Fe to Te ratio was fixed to $1.087: 1$ with the excess iron located statistically in the $2 c$ $\left(1 / 4 \frac{1}{4} Z_{\mathrm{Fe} 2}\right)$ position. Fig. 1 shows the unit cell of $\mathrm{Fe}_{1.087} \mathrm{Te}$. The Fe1-Te bond length is 2.603(1) $\AA$ and the two independent Te-Fe1-Te angles are 94.53(4) and 117.42(9) ${ }^{\circ}$, respectively, which indicates a substantial deviation from ideal tetrahedral angle of $109.5^{\circ}$. The Te-Fe1-Te angles of $94.53^{\circ}$ are located on planes parallel to the $c$-axis and the $\mathrm{FeTe}_{4}$ tetrahedra are elongated along the $c$-axis. The interstitial $\mathrm{Fe} 2$ atoms are coordinated to four Te atoms within the FeTe layer with bonds 
of 2.7106(9) $\AA$ plus an additional shorter bond of 2.56(1) $\AA$ to Te in the neighbouring FeTe layer. The Fe1-Fe1 and Fe1-Fe2 distances within the FeTe layers are 2.7035(1) and 2.739(9) A, respectively. These distances are relatively short and allow for some interaction between the interstitial $\mathrm{Fe} 2$ atoms and the Fe1 atoms within the FeTe layers. However, iron metal-metal bonds are in contrast to many other transition metals somewhat controversial, and the above-mentioned $\mathrm{Fe}-\mathrm{Fe}$ distances are in the upper end of what is normally considered to be the range for $\mathrm{Fe}-\mathrm{Fe}$ bonds (in e.g. iron carbonyls, 2.46 to $2.78 \AA$ [19], and the shortest Fe-Fe distance in elemental iron is $2.485 \AA$ ).

The high-pressure X-ray diffraction measurements on $\mathrm{Fe}_{1.087} \mathrm{Te}$ were performed in the pressure range from 0.0001 to $20 \mathrm{GPa}$, and selected powder patterns are shown in Fig. 2. Inspection of the recorded high-pressure data for $\mathrm{Fe}_{1.087} \mathrm{Te}$ revealed systematic broadening of the Bragg peaks for pressures higher than $\approx 12 \mathrm{GPa}$ and the sample was found to become amorphous at pressures higher than $16.6 \mathrm{GPa}$. The crystalline $\mathrm{Fe}_{1.087} \mathrm{Te}$ phase was partly recovered after decompression as seen from the upper pattern in Fig. 2. The recorded powder patterns did not indicate any change of symmetry within the investigated pressure range, and the refinements of lattice parameters were therefore done assuming tetragonal symmetry at all measured pressures. The pressure dependence of the lattice parameters $a$ and $c$ are shown in Fig. 3. The $c$-axis is found to be the more compressible and the compressibility of this axis is furthermore seen to decrease at $\approx 3.7 \mathrm{GPa}$. An additional change in the compressibility of the $c$-axis is observed at about $10 \mathrm{GPa}$ where the compressibility is seen to increase dramatically. In contrast, the length of the $a$-axis decreases smoothly as the pressure increases. The $c / a$-ratio is plotted as a function of pressure in the inset graph of Fig. 3 and it is seen to exhibit a minimum at $\approx 3.7 \mathrm{GPa}$ and a steep decrease at $10 \mathrm{GPa}$. Within the pressure range from ambient pressure to $3.7 \mathrm{GPa}, \mathrm{Fe}_{1.087} \mathrm{Te}$ shows almost the same degree of anisotropic compression $(\Delta a / a=2.36 \%$ and $\Delta c / c=3.31 \%)$ as $\mathrm{BaFe}_{2} \mathrm{As}_{2}$ [20]. The observed decrease in compressibility of the $c$-axis at $\approx 3.7 \mathrm{GPa}$ corresponds well to what has been observed for $\mathrm{Fe}_{1.05} \mathrm{Te}$. The $c$-axis of this compound was found to decrease by $5 \%$ in the pressure range from ambient to $\approx 4 \mathrm{GPa}$ and becoming less compressible above this pressure, see below for further discussion [21]. The changes in compressibility of the $c$-axis might reflect changes in the degree of distortion of the $\mathrm{FeTe}_{4}$ tetrahedra, but structural refinements at high pressures are required to clarify this issue. The fact that the compressibility of the $c$-axis increases at $\approx 10 \mathrm{GPa}$ is rather interesting. Takahashi et al. proposed based on resistivity measurements the existence of two low-temperature 
high-pressure phases HP I and HP II, and extrapolation of phase boundary of the HP II phase indicated that it should be stable at $300 \mathrm{~K}$ at a pressure of $\approx 12 \mathrm{GPa}$ [14]. It is therefore likely that the increased compressibility of the $c$-axis observed at $\approx 10 \mathrm{GPa}$ is related to the existence of the HP II phase. A high-pressure low temperature neutron diffraction study is planned to clarify this issue.

A comparison of the high-pressure behaviours of $\mathrm{Fe}_{1.087} \mathrm{Te}, \mathrm{BaFe}_{2} \mathrm{As}_{2}$ and $\mathrm{FeSe}$ shows that they are different. $\mathrm{BaFe}_{2} \mathrm{As}_{2}$ undergoes at ambient temperature a tetragonal to orthorhombic phase transition at $16.5 \mathrm{GPa}$ [22]. The low temperature orthorhombic phase of this compound observed at ambient pressure is suppressed at $\approx 1.2 \mathrm{GPa}$ and no structural change is observed upon cooling in the pressure range $1.2<P \leq 6 \mathrm{GPa}$ [20,23]. Different high-pressure behaviours have been observed for FeSe at ambient temperature. Stemshorn et al. report a transition from the tetragonal $P 4 / \mathrm{nmm}$ structure to a hexagonal NiAs-type structure at $10 \pm 2 \mathrm{GPa}$ and the transition is accompanied by a volume collapse of $16 \%$. The hexagonal NiAs-type structure was found to be stable up to a pressure of at least $40 \mathrm{GPa}$. In addition a transition to an amorphous phase was observed in the pressure range from 15 to $40 \mathrm{GPa}$ at $10 \mathrm{~K}$ [24]. Kumar et al. report that FeSe transforms to an orthorhombic Pbnm structure at $11 \mathrm{GPa}$ at ambient temperature. This phase was found to be stable up to the maximum measured pressure of $33 \mathrm{GPa}$. Compression at $8 \mathrm{~K}$ showed a distortion of the lowtemperature Cmma phase and the appearance of a high-pressure Pbnm phase at $\approx 1.6 \mathrm{GPa}$. The orthorhombic phase becomes the major phase above $9 \mathrm{GPa}$ and a mixed-phase region exists up to $26 \mathrm{GPa}$ and no transition to an amorphous phase was observed up to $31 \mathrm{GPa}$ in this study [25].

Fig. 4 shows the unit-cell volume of $\mathrm{Fe}_{1.087}$ Te plotted as function of pressure. The third-order BirchMurnaghan (B-M) equation of state

$$
P=\frac{3}{2} B_{0}\left(x^{-7 / 3}-x^{-5 / 3}\right)\left[1-\frac{3}{4}\left(4-B_{0}^{\prime}\right)\left(x^{-2 / 3}-1\right)\right]
$$

where $x=V / V_{0}$ where $V$ and $V_{0}$ is the volume at pressure $P$ and zero pressure, respectively, was used for the determination of the zero pressure bulk modulus $B_{0}$ and its pressure derivative $B_{0}{ }^{\prime}$ [26]. The changes above described in the compressibility of the $c$-axis combined with the fact that trial fits of the B-M equation of state in the pressure range $0 \leq P \leq 10 \mathrm{GPa}$ gave unrealistic values of $B_{0}$ and $B_{0}{ }^{\prime}$ suggest that the fitting should be done in different pressure regions. A least-squares fit to the measured $P V$-data in the pressure range $0 \leq P<5 \mathrm{GPa}$ yielded $B_{0}=36(1) \mathrm{GPa}$ and $V_{0}$ was 
treated as a refinable parameter and the fit yielding $V_{0}=92.4(2) \AA^{3}$. The second fit was done in the pressure range $5<P \leq 10 \mathrm{GPa}$ yielding $B_{0}=88(5) \mathrm{GPa}$ and $V_{0}=87.5(3) \AA^{3}$ and $B_{0}{ }^{\prime}$ was fixed at the value of 4 in both fits. The results are in good agreement with the results obtained in a recent highpressure X-ray and neutron diffraction study of $\mathrm{Fe}_{1.05} \mathrm{Te}$. This study showed the existence of a tetragonal T-phase with $B_{0}=31(1) \mathrm{GPa}$ below $4 \mathrm{GPa}$ and a collapsed tetragonal phase, the cT-phase with $B_{0}=88.7(4) \mathrm{GPa}$ above this pressure [21]. From Fig. 4 it is seen that the transition from the Tto the cT-phase in $\mathrm{Fe}_{1.087} \mathrm{Te}$ takes place at $\approx 4.1 \mathrm{GPa}$. No attempt was made in the present study to fit data recorded above $10 \mathrm{GPa}$ as data quality here is considerably lower. However, our data indicate the existence of an additional collapsed tetragonal phase cT' above $10 \mathrm{GPa}$.

The low values of the bulk moduli of the T-phases of $\mathrm{Fe}_{1+x} \mathrm{Te}$ for $x=0.087$ and 0.05 show that they are soft materials. For comparison, the bulk modulus of FeSe has been determined to 33 and $31 \mathrm{GPa}$ at 50 and $190 \mathrm{~K}$, respectively [27], and no collapsed tetragonal phase was observed for this compound [25]. Higher bulk moduli have been determined for the related iron arsenide compounds. In the case of $\mathrm{NdFeAsO}{ }_{0.88} \mathrm{~F}_{0.12}$ and optimally doped $\mathrm{LaFeAsO}_{0.89} \mathrm{~F}_{0.11}$ bulk moduli of 102 and 66 $\mathrm{GPa}$, respectively, were reported [28,29], while the bulk modulus of $\mathrm{BaFe}_{2} \mathrm{As}_{2}$ was determined as 59(2) $\mathrm{GPa}[20]$. The chalchogenides FeSe and $\mathrm{Fe}_{1+\mathrm{x}} \mathrm{Te}$ are clearly more compressible than the iron pnictides, and the larger compressibility is presumably also responsible for the large value of $\mathrm{d} T_{c} / \mathrm{d} P$ observed for FeSe [4]. The T- and cT-phases observed for $\mathrm{Fe}_{1+x} \mathrm{Te}$ resemble to some degree the high-pressure behaviour of $\mathrm{CaFe}_{2} \mathrm{As}_{2}$ which at $0.63 \mathrm{GPa}$ transforms from a tetragonal hightemperature phase to a "collapsed" tetragonal low-temperature phase in the temperature range from 150 to $200 \mathrm{~K}$, in which the $c$-axis is reduced from 11.6 to $10.5 \AA$ upon cooling, while the $a$-axis is simultaneously elongated by $\approx 0.07 \AA$ [30]. However, the T-phase of $\mathrm{CaFe}_{2} \mathrm{As}_{2}$ has an estimated zero pressure bulk modulus of $60 \mathrm{GPa}$ at $50 \mathrm{~K}$ and it transforms abruptly into the collapsed cTphase at $0.3 \mathrm{GPa}$ at this temperature, undergoing a volume reduction of $5 \%$. In contrast, no discontinuous volume change is observed in the case of $\mathrm{Fe}_{1+x} \mathrm{Te}$ at the transition from the T-phase to the cT-phase, and the transition also takes place at a considerably higher pressure than in the case of $\mathrm{CaFe}_{2} \mathrm{As}_{2}$. Interestingly, $\mathrm{NdFeAsO}_{0.88} \mathrm{~F}_{0.12}$ also exhibits a continuous isostructural phase transition at $\approx 10 \mathrm{GPa}$, thereby resembling the high-pressure behaviour of $\mathrm{Fe}_{1+x} \mathrm{Te}$. The highpressure phase of this compound was found to have a substantially higher bulk modulus, $B_{0}=$ 245(9) GPa [28]. This kind of isostructural phase transition may be common in iron-based superconductors and structurally related iron chalchogenides, and studies of the atomic structure 
evolution upon compression might provide essential information for the understanding of the pressure dependence of the superconducting transition temperature. The mechanism of the isostructural transitions has not been clarified, but first principle studies of $\mathrm{CaFe}_{2} \mathrm{As}_{2}$ have shown that the magnetic moment of iron is drastically reduced in the collapsed tetragonal phase, and the As-As bonds between neighboring FeAs layers are enhanced at high pressure [31]. A spin state change of $\mathrm{Fe}$ in $\mathrm{Fe}_{1+x} \mathrm{Te}$ has therefore also been suggested as the reason for the appearance of the cT-phase in this compound [21].

\section{Conclusion}

It has been shown that $\mathrm{Fe}_{1.087} \mathrm{Te}$ exhibits three phases in the pressure range from ambient to 16.6 $\mathrm{GPa}$ and becomes amorphous above this pressure. All three phases have tetragonal symmetry and the two high-pressure phases labelled cT and cT' are stable in the pressure ranges $0 \leq P<4.1 \mathrm{GPa}$ and $4.1<P \leq 10 \mathrm{GPa}$, respectively. The zero pressure bulk moduli of the $\mathrm{T}$ and $\mathrm{cT}$ phases were found to be 36(1) and 88(5) $\mathrm{GPa}$, respectively, showing that the low pressure phase of $\mathrm{Fe}_{1.087} \mathrm{Te}$ is relatively soft. The presence of the $\mathrm{cT}$ and $\mathrm{cT}$ ' phases are possibly related to pressure-induced changes of the spin state of iron or changes in bonding between neighbouring FeTe layers. Further elucidation of this issue will require studies of the crystal structure at high pressure in combination with electronic structure calculations.

\section{Acknowledgements}

The authors thank HASYLAB-DESY for permission to use the synchrotron radiation facility, and DANSCATT is gratefully acknowledged for financial support. 


\section{References}

[1] F.C. Hsu, J.Y. Luo, K.W. The, T.K. Chen, T.W. Huang, P.M. Wu, Y.C. Lee, Y.L. Huang, Y. Y. Chu, D.C. Yan, M.K. Wu, Superconductivity in the PbO-type structure $\alpha-F e S e$, Proc. Nat. Acad. Sci. 105 (2008) pp. 14262-14264.

[2] Y. Kamihara, T. Watanabe, M. Hirano, H. Hosono, Iron-Based Layered Superconductor La $\left[O_{1-x} F_{x}\right] F e A s(x=0.05-0.12)$ with $T_{c}=26$ K, J. Am. Chem. Soc. 130 (2008) pp. 32963297.

[3] H. Takahashi, K. Igawa, K. Arii, Y. Kamihara, M. Hirano, H. Hosono, Superconductivity at $43 \mathrm{~K}$ in an iron-based layered compound $\mathrm{LaO}_{1-x} F_{x} F e A s$, Nature 453 (2008) pp. 376-378.

[4] Y. Mizuguchi, F. Tomioka, S. Tsuda, T. Yamaguchi and Y. Takan, Superconductivity at 27 K in tetragonal FeSe under high Pressure, Appl. Phys. Lett. 93 (2008) 152505(1)-152505(3).

[5] D. Fruchart, P. Converts, P. Wolfers, R. Madar, J. P. Senateur, R. Fruchart, Structure Antiferromagneticque de $\mathrm{Fe}_{1.125} \mathrm{Te}$ Accompagnee d'un Deformation Monoclinic, Mat. Res. Bull. 10 (1975) pp. 169-174.

[6] Y. Mizuguchi, F. Tomioka, S. Tsuda, T. Yamaguchi, Y. Takano, FeTe as a candidate material for new iron-based superconductor, Physica C 469 (2009) 1027-1029.

[7] M.H. Fang, H.M. Pham, B. Qian, T.J. Liu, E.K. Vehstedt, Y. Liu, L. Spinu, and Z.Q. Mao, Superconductivity close to magnetic instability in Fe(Se $\left.{ }_{1-x} T e_{x}\right)_{0.82}$, Phys. Rev. B 78 (2008) pp. 224503(1)-224503(5).

[8] N. Stojilovic, A. Koncz, L.W. Kohlman, Rongwei Hu, C. Petrovic, S.V. Dordevic, Normal state charge dynamics of $\mathrm{Fe}_{1.06} \mathrm{Te}_{0.88} \mathrm{~S}_{0.14}$ superconductor probed with infrared spectroscopy, Phys. Rev. B 81 (2010) pp. 174518(1)-174518(5).

[9] H. Ipser, K. L. Komarek, H. Mikler, Transition Metal-Chaleogen Systems, V.: The IronTellurium Phase Diagram, Monatshefte für Chemie 105 (1974) pp. 1322-1434.

[10] H. Okamoto, L.E. Tanner, The Fe-Te (Iron-Tellurium) System, Bull. Alloy Phase Diagrams 11 (1990) pp. 371-376.

[11] F. Grønvold, H. Haraldsen, J. Vihovde, Phase and Structural Relations in the System Iron Tellurium, Acta Chem. Scand. 8 (1954) pp. 1927-1942.

[12] Wei Bao, Y. Qiu, Q. Huang, M.A. Green, P. Zajdel, M.R. Fitzsimmons, M. Zhernenkov, S. Chang, Minghu Fang, B. Qian, E. K. Vehstedt, Jinhu Yang, H. M. Pham, L. Spinu, and Z. Q. Mao, Tunable $(\delta \pi, \delta \pi)$-Type Antiferromagnetic Order in $\alpha-F e(T e, S e)$ Superconductor, PRL 102 (2009) pp. 247001(1)-247001(4). 
[13] J.W. Lynn, P. Dai, Neutron studies of the iron-based family of high $T_{C}$ magnetic superconductors Physica C 469 (2009) 469-476.

[14] H. Takahashi, H Okada, H. Takahashi, Y. Mizuguchi, Y. Takano, Electrical resistivity measurements under high pressure for FeTe.92, J. of Phys.: Conference Series 200 (2010) pp. 012196(1)-012196(4).

[15] J.S. Olsen, Developments and new possibilities in high pressure powder diffraction with synchrotron radiation. Results for cerium metal and $U_{6} F e$, Rev. Sci. Instrum. 63 (1992) pp. 1058-1061.

[16] G. Huber, K. Syassen, W.B. Holzapfel, Pressure dependence of 4 fevels in europium pentaphosphate up to 400 kbar, Phys. Rev. B15 (1977) pp. 5123-5128.

[17] H. K. Mao, J. Xu, and P. M. Bell, Calibration of the ruby pressure gauge to 800-kbar under quasi-hydrostatic conditions, J. Geophys. Res.-Solid Earth and Planets 91 (1986), pp. 4673 4676.

[18] R. Viennois, E. Giannini, D. van der Marel, R. Černý, Effect of Fe excess on structural, magnetic and superconducting properties of single-crystalline $\mathrm{Fe}_{1+x} \mathrm{Te}_{1-y} \mathrm{~S} e_{y}$, J. Solid State Chem. 183 (2010) pp. 769-775.

[19] L. Pauling, Metal-metal bond lengths in complexes of transition metals, Proc. Natl. Acad. Sci. USA, 73 (1976) pp. 42904293.

[20] J.-E. Jørgensen, T. C. Hansen, High-pressure neutron diffraction study of $\mathrm{BaFe}_{2} \mathrm{As}_{2}$, Eur. Phys. J. B 78 (2010). pp. 411-415.

[21] C. Zhang, W. Yi, L. Sun, X.-J. Chen, R.J. Hemley, H.-K. Mao, W. Lu, X. Dong, L. Bai, J. Liu, A.F.M.D. Santos, J.J. Molaison, C.A. Tulk, G. Chen, N. Wang, Z. Zhao, Pressureinduced lattice collapse in the tetragonal phase of single-crystalline $F_{1.05} T e$, Phys. Rev. B 80 (2009) pp. 144519(1)-144519(5).

[22] J.-E. Jørgensen, J. Staun Olsen, L. Gerward, On the compressibility of $\mathrm{BaFe}_{2} \mathrm{As}_{2}$, Solid State Commun. 149 (2009) pp. 1161-1163.

[23] S.A.J. Kimber, A. Kreyssig, Y.-Z. Zhang, H. O. Jeschke, R. Valentí, F. Yokaichiya, E. Colombier, J. Yan, T. C. Hansen, T. Chatterji, R.J. McQueeney, P.C. Canfield, A. I. Goldman, D. N. Argyriou, Similarities between structural distortions under pressure and chemical doping in superconducting $\mathrm{BaFe}_{2} \mathrm{As}_{2}$, Nature Materials 8 (2009) pp. 471-475. 
[24] A.K. Stemshorn, G. Tsoi, Y.K. Vohra, S. Sinogeiken, P.M. Wu,Y. Huang, S.M. Rao, M.-K. $\mathrm{Wu}, \mathrm{K} . \mathrm{W}$. Yeh, Low temperature amorphization and superconductivity in FeSe single crystals at high pressures, J. Mater. Res. 25 (2010) pp. 396-400.

[25] R.S. Kumar, Y. Zhang, S. Sinogeikin,Y. Xiao, S. Kumar, P. Chow, A.L. Cornelius, C. Chen, Crystal and Electronic Structure of FeSe at High Pressure and Low Temperature, J. Phys. Chem. B 114 (2010) pp. 12597-12606.

[26] F. Birch, Finite Strain Isotherm and Velocities for Single-Crystal and Polycrystalline NaCl at High-Pressures and $300^{\circ}$ K, J. Geophys. Res. 83 (1978) pp. 1257-1268.

[27] J. N. Millican, D. Phelan, E. L. Thomas, J. B. Leão, E. Carpenter, Pressure-induced effects on the structure of the FeSe superconductor, Solid State Comm. 149 (2009) pp. 707-710.

[28] J. Zhao, L. Wang, D. Dong, Z. Liu, H. Liu, G. Chen, D. Wu, J. Luo, N. Wang, Y. Yu, C. Jin, and Q. Guo, Struture Stability and Compressibility of Iron-Based Superconductor $N d\left(O_{0.88} F_{0.12}\right) F e A s$ under High Pressure, J. Am. Chem. Soc., 130 (2008) pp. 13828-13829.

[29] H. Takahashi, H. Okada, K. Igawa, K. Arii, Y. Kamihara, S. Matsuishi, M. Hirano, H. Hosono, K. Matsubayashi, Y. Uwatoko, High-pressure Studies on Superconducting ironbased LaFeAsOl-xFx, LaFePO and $\mathrm{SrFe}_{2} \mathrm{As}_{2}$, J. Phys. Soc. Jpn. 77, Suppl. C (2008) pp. 7883.

[30] A. Kreyssig, M. A. Green, Y. Lee, G. D. Samolyuk, P. Zajdel, J.W. Lynn, S.L. Bud'ko, M.S. Torikachvili, N. Ni, S. Nandi, J.B. Leão, S. J. Poulton, D.N. Argyriou, B.N. Harmon, R.J. McQueeney, P.C. Canfield, A.I. Goldman, Pressure-induced volume-collapsed tetragonal phase of $\mathrm{CaFe}_{2} \mathrm{As}_{2}$ as seen via neutron scattering Phys. Rev. B 78 (2008) pp. 184517(1)-184517(6)

[31] T. Yildirim, Strong Coupling of the Fe-Spin State and the As-As Hybridization in IronPnictide Superconductors from First-Principle Calculations, PRL 102 (2009) pp. 37003(1)37003(4). 


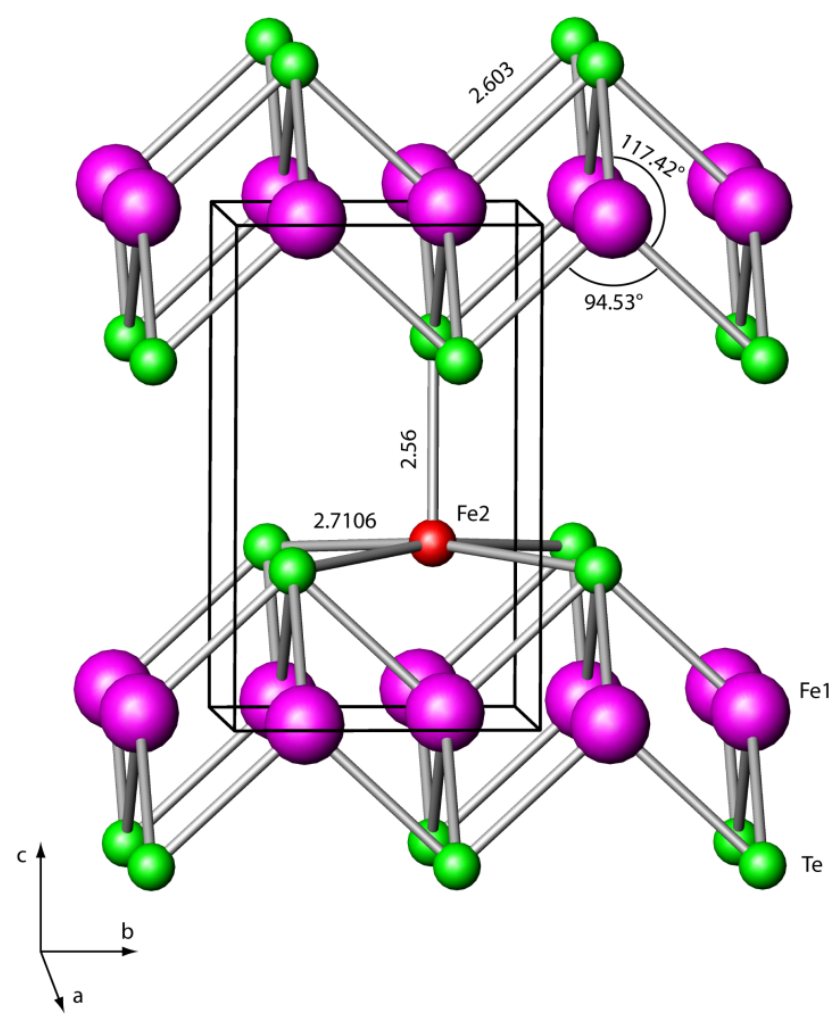

Fig. 1. Crystal structure of $\mathrm{Fe}_{1+x} \mathrm{Te}$ showing the FeTe layers composed of edge sharing $\mathrm{FeTe}_{4}$ tetrahedra. Only one interstitial Fe atom (labeled Fe2) is shown for clarity, and selected bond lengths are given in $\AA$. The unit cell is shown with thin solid lines.

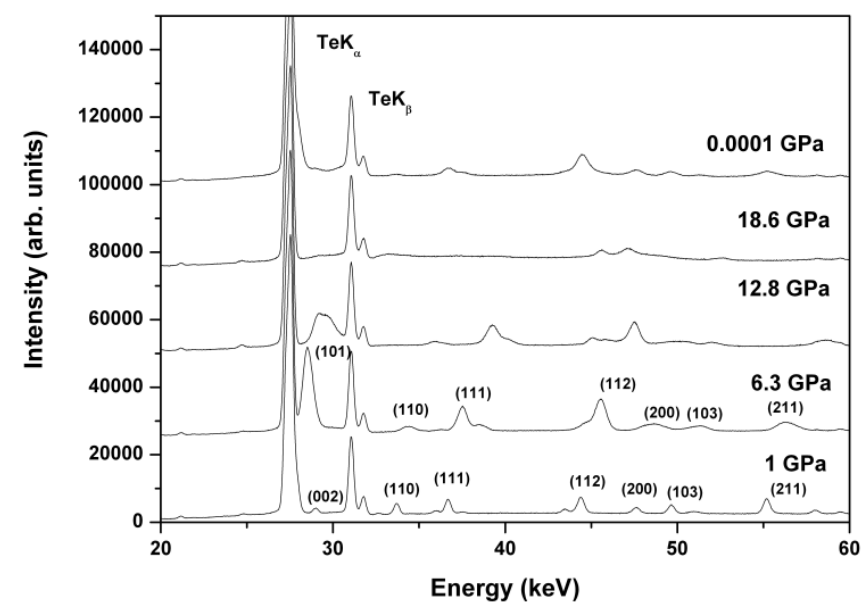

Fig. 2. Selected high-pressure energy dispersive $X$-ray powder patterns of $\mathrm{Fe}_{1.085} \mathrm{Te}$. The upper pattern recorded at ambient conditions was measured after decompression. The Miller indices for the Bragg peaks of the $\mathrm{Fe}_{1.085} \mathrm{Te}$ phase are shown on the 1 and $6.3 \mathrm{GPa}$ patterns while the $\mathrm{K}_{\alpha}$ and $\mathrm{K}_{\beta}$ fluorescence lines of Te are marked on the upper pattern. The (101) reflection is coinciding with the Te $\mathrm{K}_{\alpha}$ line in the $1 \mathrm{GPa}$ pattern and the weak (002) reflection is not visible in $6.3 \mathrm{GPa}$ pattern. 


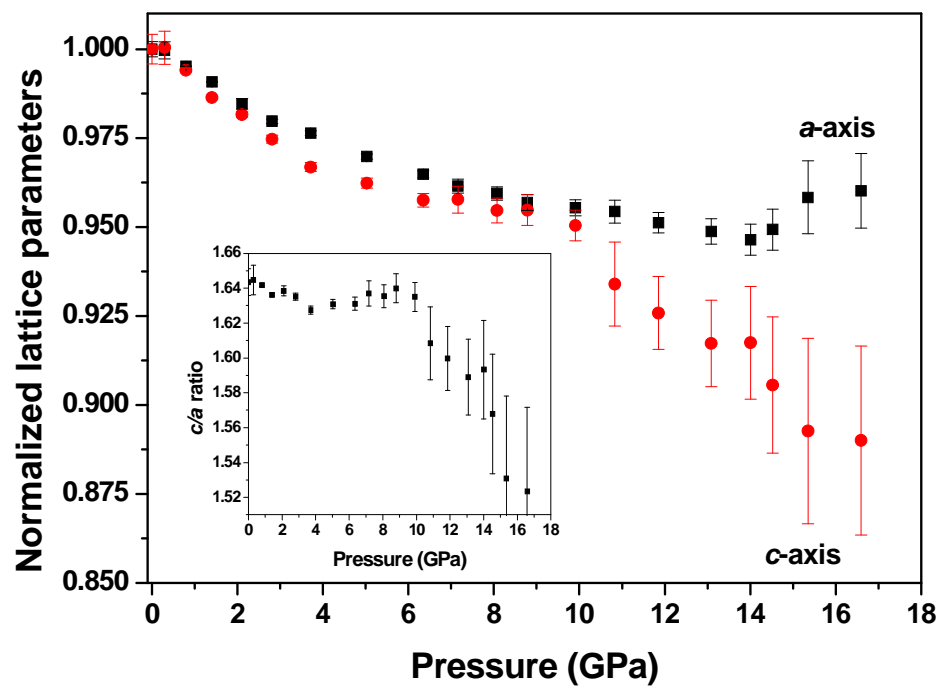

Fig. 3. Lattice parameters $a$ and $c$, normalized to their ambient pressure values plotted as a function of pressure. The compressibility of the $c$-axis is seen to decrease at $\approx 3.7 \mathrm{GPa}$ and increase dramatically at $\approx 9 \mathrm{GPa}$. The inset graph shows the $c / a$ ratio plotted as a function of pressure. 


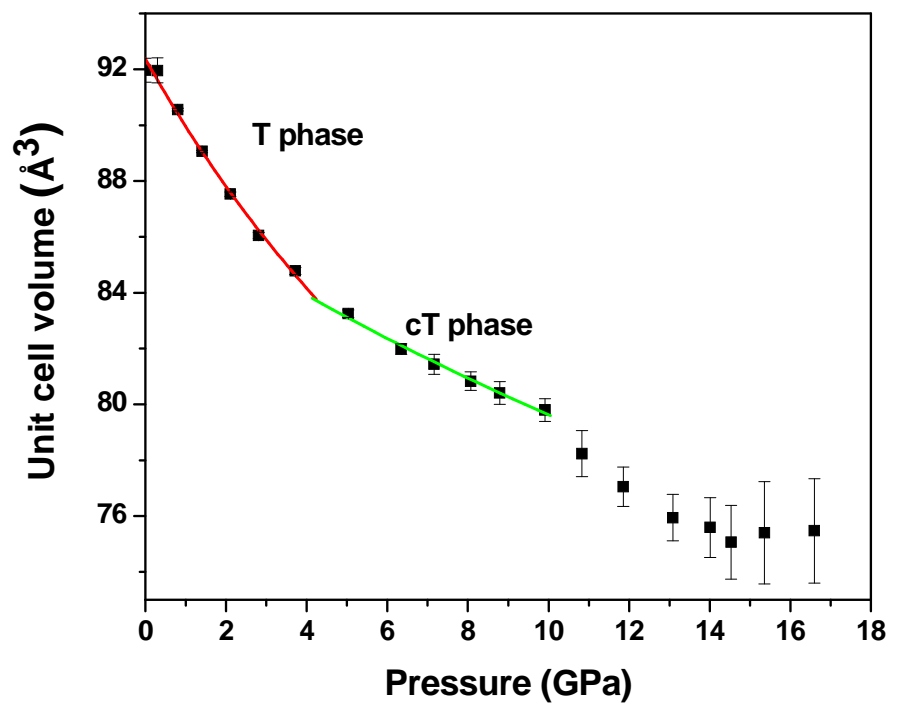

Fig. 4. The unit cell volume plotted as a function of pressure. The solid lines represents fits of the third-order Birch-Murnaghan equation to the experimental data points in the T-and cT-phases as described in the text. 